\title{
Behavior of Adsorbed Albumin Film on CoCrMo Alloy under In-situ Observation
}

\author{
Kazuhiro Nakashima $^{1,2)^{*}}$, Yoshinori Sawae ${ }^{1,2)}$, Teruo Murakami ${ }^{2)}$ and Stefano Mischler ${ }^{3)}$ \\ ${ }^{1)}$ Department of Mechanical Engineering, Kyushu University \\ 744 Motooka, Nishi-ku, Fukuoka 819-0395, Japan \\ ${ }^{2)}$ Research Center for Advanced Biomechanics, Kyushu University \\ 744 Motooka, Nishi-ku, Fukuoka 819-0395, Japan \\ ${ }^{3)}$ Tribology and Interfacial Chemistry Group, École Polytechnique Fédérale de Lausanne \\ 1015 Lausanne, Switzerland \\ *Corresponding author: nakaji@mech.kyushu-u.ac.jp
}

( Manuscript received 26 December 2013; accepted 24 March 2015; published 15 April 2015 )

( Presented at Technical Session in the 5th World Tribology Congress TORINO September 2013)

\begin{abstract}
The protein film adsorbed on joint prosthesis material affects tribological property. It is expected that optimum protein film structure could improve friction and wear of joint prosthesis materials. Therefore, it is important to understand and to clarify the effect of protein film on tribological property. In the rubbing combination of ultra-high molecular weight polyethylene (UHMWPE) and CoCrMo alloy, the adsorption behavior of bovine serum albumin (BSA) and its effect on frictional property during friction were investigated by using electrochemical method. Friction force and electric potential were measured under open circuit potential (OCP) condition, but friction force and electric current were measured under $-0.2 \mathrm{~V}$ applied potential condition. Under OCP condition, adsorbed BSA was peeled off at first rubbing, after that BSA re-adsorbed on surface under shear force condition. BSA adsorbed film reconstructed by shear force was sustained during rubbing. It is considered that the conformation of adsorbed BSA under shear stress was changed from that of static adsorption, because readsorbed BSA molecule did not show exfoliation from metal surface, which means that adsorption strength was increased from static adsorption. Under applied potential condition where wear performance of BSA film is expected to be evaluated, BSA showed the desorption at first rubbing, and gradual decrease in desorption amount. Therefore, it is confirmed that BSA film on metal surface under rubbing condition is reconstructed during rubbing. The adsorbed film formed during rubbing is stable and has an optimally-adapted structure for shear force and can sustain its property during rubbing.
\end{abstract}

Keywords: boundary lubrication, natural synovial fluid constituents, protein adsorption, electrochemical method, bovine serum albumin, ultra-high molecular weight polyethylene, CoCrMo alloy, joint prosthesis material.tribology, design, maintenance, trouble, case study, bearing, seal

\section{Introduction}

The combination of metal materials and ultrahigh molecular weight polyethylene (UHMWPE) has been successfully used in joint prostheses for a long time. However, the longevity of such implants is limited by the wear of UHMWPE. The friction and wear properties of UHMWPE are affected among other parameters by the chemistry of periprosthetic synovial fluid $[1,2]$ and in particular by proteins and phospholipids. Long immersion periods in the synovial fluid change the mechanical properties of UHMWPE [3,4]. Proteins and phospholipids affect the corrosion and the surface chemistry of the metallic alloy [5,6]. Under rubbing, proteins and phospholipids adsorb on the material surfaces, and prevent direct touching of two rubbing surfaces. The adsorbed film plays an important role as boundary layer for friction and wear properties. Therefore, it is important to understand the effect of constituents in periprosthetic synovial fluid on property of joint prosthesis materials. In addition, when the synovia constituents adsorb on material surfaces, it is considered that constituents form composite structure, so that the consideration about competitive adsorption, multi-stratification and film structuring of adsorbed constituents for friction and wear phenomena is 
necessary. The adsorbed film practically affected tribological property [1]. To clarify the effect of proteins, adsorbed protein film was observed using by fluorescent method [7]. From the observation, two types of adsorbed protein film structures of coexisting bovine serum albumin (BSA) and human $\gamma$-globulin (HGG), were observed. The adsorbed protein film, which resulted in low friction and low wear, showed a lamellar structure composed from BSA layer and HGG layer. Another protein film structure, which increased friction and wear, showed a heterogeneous structure of proteins. The lamellar structure can slip at boundary of protein layers, so that it is considered that lamellar structure showed low friction and low wear. The results clearly indicate that protein film can control frictional property. If the adsorbed protein film formation could be controlled, it is considered that tribological property of joint prosthesis can be improved by optimal protein film.

There are many studies on static protein adsorption on artificial materials, but, there is a little study on protein adsorption under rubbing conditions [8-11]. Static protein adsorption is affected by surface hydrophilicity of artificial material, electrical property of artificial material, protein conformation, protein concentration, solvent $\mathrm{pH}$, temperature and so on. When few types of protein were added into solution, each protein additive ratio and competitive adsorption should be considered. Under rubbing condition, it must be considered on additional terms such as, shear stress by frictional force, local heating, and electrical charge change by friction.

It is reported that rubbing affects protein conformation due to shearing and heating during rubbing $[9,10,12]$. It was shown that conformational change has a great effect on frictional property. Mishina et al. showed albumin in lubricant after friction test had become low molecular weight [9]. Heuberger et al. showed albumin adsorbed on worn surface changes conformation and that affects frictional behavior [10]. The protein conformational change induces different interaction between protein molecules and material surface and between adsorbed protein molecules and protein molecules dissolved in lubricant. The different interaction affects adsorbed protein film formation. Therefore adsorbed film composed of denatured proteins changed friction property.

In the previous studies [11,13], the behavior of adsorbed proteins was investigated through observations after frictional tests that do not account for the dynamic behavior during rubbing. Yarimitsu et al. [8] reported in-situ observation results using fluorescent technique, and they showed that proteins do adsorb on worn surfaces during rubbing. Their loading and sliding condition was too mild compared with practical joint prosthesis, because of the limitation in contact geometry related to the presence of a microscope for clear observation. The current study is initiated with the aim to evaluate the behavior of adsorbed proteins during rubbing under clinically relevant conditions for joint prostheses.

It should be taken into consideration that not only conformation change but also electric property can have effects on protein adsorption. Electrolyte affects surface electric property [5,6,14-16]. In addition, two rubbing surfaces also changes material potential. Joint prosthesis is rubbed in synovial fluid, so that the surface potential will be changed during walking. It is considered that different potential affects differently protein adsorption and forms distinct adsorbed protein film structure. That will induce different tribological property. To understand the potential during rubbing, electrochemical method is suitable, because the method allows to measure potential transition in-situ. The electrochemical studies on joint prosthesis are mainly focused on metal-on-metal joints. In those cases, many studies are focusing wear of materials. The electrochemical method can measure surface reduction behavior, so that it allows to measure passive film and bulk metal loss induced by wear. In the combination of metal-on-UHMWPE, surface reduction effect indicates wear of surface zone which includes adsorbed protein film or contamination layer under less wear condition. Therefore, adsorption and desorption behavior of protein molecules can be measured under in-situ observation.

In this study, the behavior of adsorbed protein film on CoCrMo alloy during rubbing was observed using electrochemical technique in order to obtain in-situ, real time information on adsorption and desorption of proteins.

\section{Experimental methods}

Sliding pair of UHMWPE (GUR1050) pin specimen with spherical end (10 $\mathrm{mm}$ diameter) and CoCrMo alloy (ASTM F75) disk with $5 \mathrm{~mm}$ thick and $25 \mathrm{~mm}$ diameter as a work electrode (WE) was tested in a reciprocating pin-on-disk tribometer equipped with electro-chemical

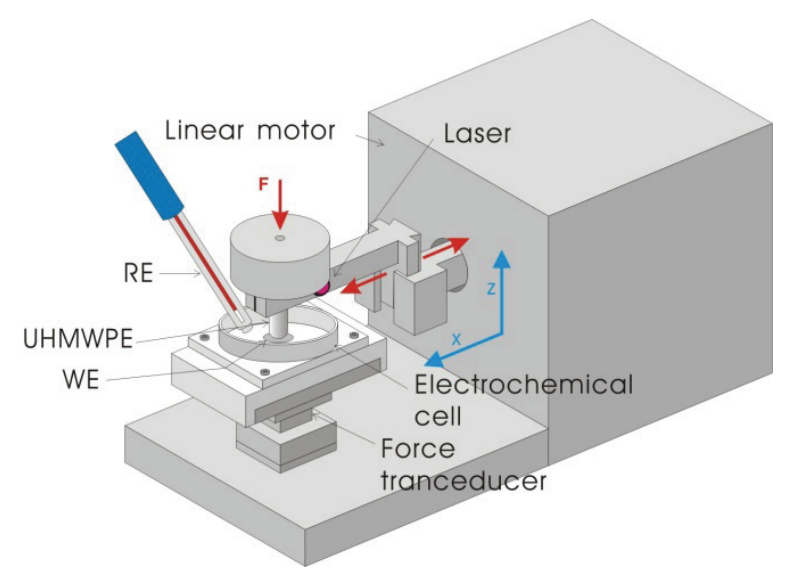

Fig. 1 Reciprocating pin-on-disk tribometer with electrochemical cell 
cell for potential and current measurement shown in Fig. 1. The applied load was $6.54 \mathrm{~N}(24.8 \mathrm{MPa}$, maximum Hertzian pressure), stroke of reciprocating was $5 \mathrm{~mm}$ and sliding speed was $10 \mathrm{~mm} / \mathrm{s}(1 \mathrm{~Hz})$. The friction test was continued for $60 \mathrm{~min}$. Lubricant employed in this study was PBS (pH 7.4) with/without BSA 0.7, 1.4 and 2.1 mass $\%$. BSA was selected because of main protein constituent usually more than $60 \%$ in periprosthetic synovial fluid. The electro-chemical cell was filled with lubricant after test specimens were set up. The potential between CoCrMo alloy and electrolyte was measured by a Calomel reference probe as reference electrode (RE). The counter electrode for current measurement was a platinum wire. The tests were carried out under OCP (open circuit potential) or $-0.2 \mathrm{~V}$ potential applied. Electric potential was measured for $10 \mathrm{~min}$ before rubbing for OCP measurement, or electric current was measured for $30 \mathrm{~min}$ before rubbing for applied potential condition. The transition of potential, frictional force and normal loading force were recorded during each rubbing test. In addition, current during the test was measured under applied potential condition. Potential or current measurement was continued for 10 min or $30 \mathrm{~min}$ after rubbing, respectively. Coefficient of friction and transition of potential were calculated.

\section{Experimental results}

Figure 2 shows an example of transition of coefficient of friction under OCP condition. Similar trend of frictional coefficient transition was obtained from other experiments. In Fig. 2, the non-zero signals before and after rubbing were derived from not friction force but offset forces due to a gap between the center of UHMWPE pin and a 3-dimensional force transducer fixed under the electrochemical cell. PBS with BSA 2.1 mass $\%$ showed higher friction compared with PBS solution. This behavior indicates BSA adsorption on CoCrMo alloy surface and it changed frictional

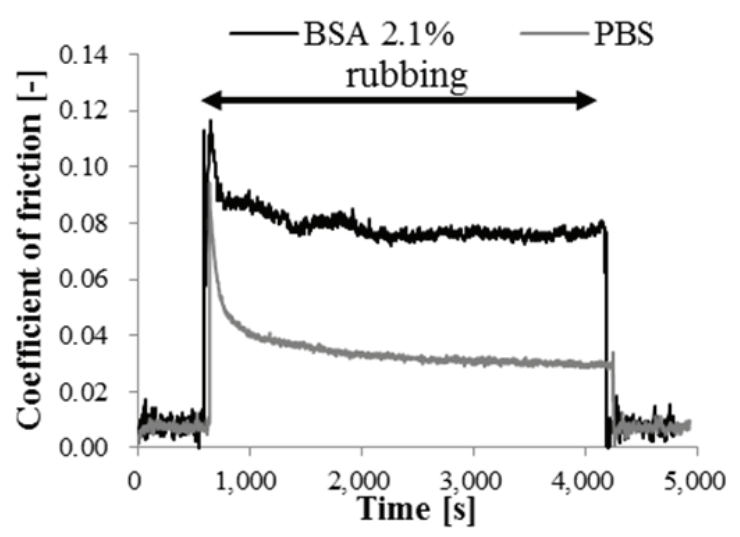

Fig. 2 Friction transition under OCP condition. At $10 \mathrm{~min}(600 \mathrm{~s})$, the rubbing test was started and continued for $60 \mathrm{~min}$

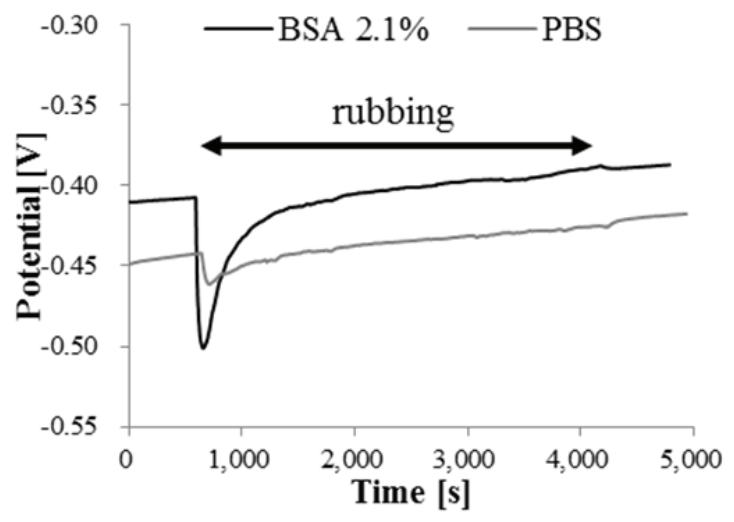

Fig. 3 Potential transition under OCP condition. At $10 \mathrm{~min}(600 \mathrm{~s})$, the rubbing test was started and continued for $60 \mathrm{~min}$

coefficient high.

The transition of potential under OCP condition is shown in Fig. 3. BSA solution showed large drop after starting of rubbing. Then, rapid increase of potential was observed. The transition in PBS was similar to that in BSA solution, although potential drop was small. It is indicated that the deoxidation brings the decrease in potential [14-16]. In this case, deoxidation of surface means physical_thinning or chemical_reduction of CoCrMo alloy surface oxide layer, such as passive film. The surface passive film can be reduced by chemical reactions, but it is difficult to expect reduction reactions of passive film of CoCrMo alloy under this mild condition, because, gradual potential increase before rubbing and after $2000 \mathrm{~s}$ during rubbing were observed shown in Fig. 3. Usually, the gradual increasing of potential transitions during rubbing indicates CoCrMo alloy surface oxidation. It is difficult to consider that potential drop or increase was caused by chemical reaction as described above. Therefore, the decrease of potential indicates physical thinning of surface oxide layer by wear of CoCrMo alloy. The surface oxide layer of CoCrMo alloy in this condition electrically includes not only metal oxide passive film, but also BSA film adsorbed on metal surface. If the potential drop was induced by wear, potential keeps low during rubbing, and potential recover takes long period $[15,16]$. Therefore, it is considered that potential transition as a drop and an increase in BSA solution indicates desorption and adsorption of BSA film, respectively. After potential drop, rapid potential increase was observed prior to gradual one. As described above, surface oxidation and deoxidation correspond to BSA adsorption and desorption on surface, respectively, and therefore, the rapid potential increase indicates BSA adsorption. It is also reported that BSA adsorbs with sliding distance [9], and the results in this study showed an effect of rubbing on BSA adsorption.

Figure 4 shows the relationship between coefficient 


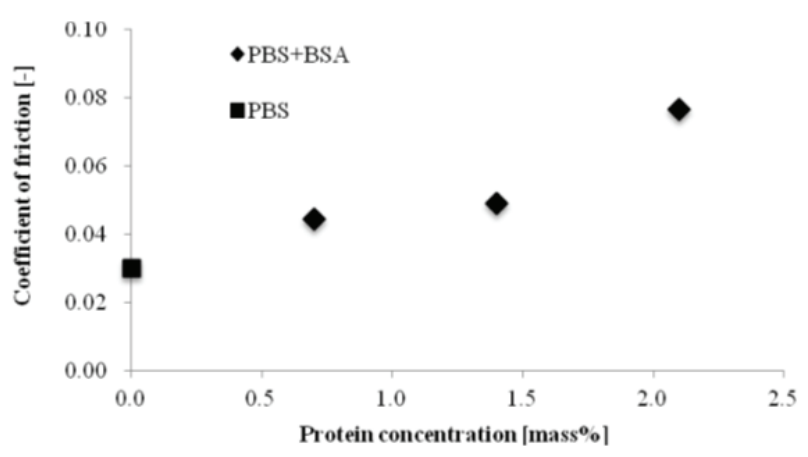

Fig. 4 Relationship between protein concentration in lubricant and coefficient of friction under OCP condition. Coefficient of friction at stable stage increases with protein concentration

of friction after friction coefficient had become stable and protein concentration of lubricant. The higher concentration showed the higher coefficient of friction. In other words, PBS without protein showed low friction. It is considered that adsorbed BSA film prevents direct contact so that it is expected that friction is reduced but the friction was increased. BSA film did not show boundary lubrication under the thin film condition.

Figure 5 shows the potential drop ( $\Delta$ potential) under OCP condition. The $\Delta$ potential is defined as the difference between initial potential before rubbing and minimum potential. The $\Delta$ potential increased with an increase of protein concentration in lubricant. In this case, the increase in $\Delta$ potential for BSA solution corresponds to amount of desorbed BSA under shear stress.

The applied potential condition was performed to investigate wear performance of BSA film. The friction coefficient under applied potential condition $-0.2 \mathrm{~V}$ are shown in Fig. 6. Coefficient of friction in BSA solution was low compared to that under OCP condition. On the other hand, that in PBS was not changed compared to that under OCP condition. Therefore, BSA adsorption had changed due to applied potential. BSA is charged negative in PBS pH 7.4. So, it may occur that adsorbed

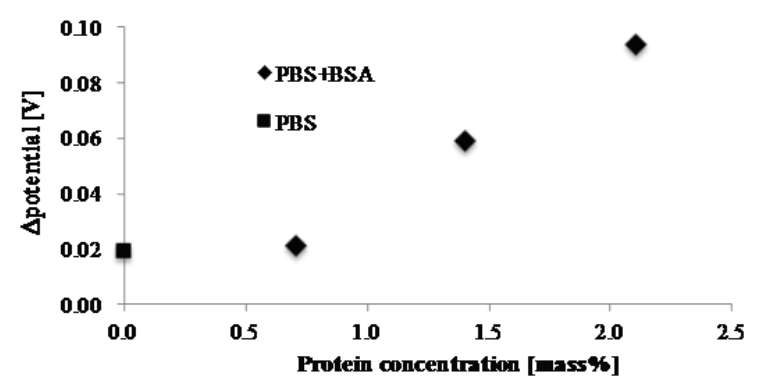

Fig. 5 Relationship between protein concentration in lubricant and $\Delta$ potential as potential drop immediately after start-up. $\Delta$ potential increases with protein concentration

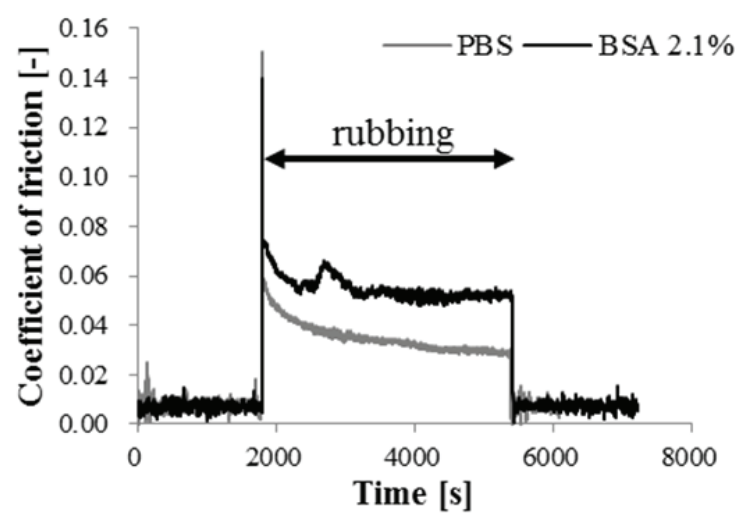

Fig. 6 Friction transition under $-0.2 \mathrm{~V}$ potential. At $30 \mathrm{~min}$ (1800 s), the rubbing test was started and continued for $60 \mathrm{~min}$

BSA molecules were decreased.

The transition of current under applied potential condition is shown in Fig. 7. The current was increased at starting of rubbing and decreased gradually with time. Current is an index of wear amount of CoCrMo alloy surface including adsorbed BSA film, therefore, the difference in current means desorption of adsorbed BSA film. The peak of current in BSA solution was higher compared to that in PBS. In considering with the results under OCP condition, readsorption of BSA should have occurred during rubbing. The difference in the current between PBS and BSA solutions indicates BSA desorption, and the current recover with time was slow for BSA solution. That means weak readsorbed BSA molecule was peeled off from the surface. The current in BSA solution under applying $-0.2 \mathrm{~V}$ potential after 4000 s was same that in PBS, so that it is considered that only bulk metal oxidation occur. As a result, adsorption and desorption of BSA molecule reached in stable state, while adsorption and desorption locally occur. In addition, coefficient of friction was high compared to that in PBS, and was stable, so that stable and strong adsorbed film under shear stress had been formed on the surface.

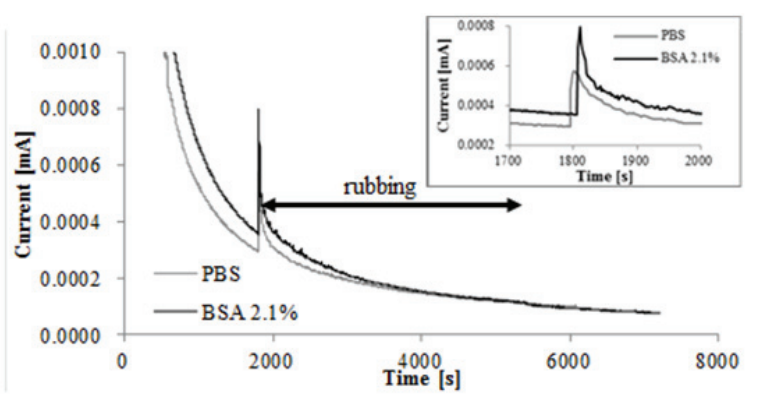

Fig. 7 Current transitions under $-0.2 \mathrm{~V}$ potential. At $30 \mathrm{~min}$ (1800 s), the rubbing test was started and continued for $60 \mathrm{~min}$ 


\section{Discussions}

In the current reciprocating test at slow speed of 10 $\mathrm{mm} / \mathrm{s}$ lubricated with PBS or BSA solution, the fluid film is estimated to be thinner than $0.2 \mu \mathrm{m}$. CoCrMo alloy surface was mirror finished, but surface roughness of UHMWPE was finish-machining and it was over $0.1 \mathrm{~mm}$ in maximum height roughness. Therefore, the lubrication mode is boundary or mixed lubrication. In thin film conditions, the adsorbed protein film can interact mutually and increases or decreases friction depending on the severity of rubbing condition.

Under OCP conditions, desorption of adsorbed protein was clearly observed by a potential drop and an abrupt friction increase. The first rubbing sheared the adsorbed protein film which statically adsorbed on material surface. The adsorbed protein film was peeled off from material surface by the first shear force shown in Fig. 3, because first rubbing showed potential drop. Then, rapid potential recover until 2,000 s was observed. As described in Experimental Results section, the recovery was due to readsorption of BSA molecules. The readsorption of BSA molecules occurred under shear stress, so that it is considered that the adsorption form or domain of BSA molecule was different from static adsorption, and the conformation would be denatured [10-12]. BSA molecule is folded hydrophilic part outside in aqueous solution. The static adsorption on metallic material surface normally occurs with facing hydrophilic domain in a protein molecule. Because BSA molecule is deformed physically under shear stress, the conformation is changed, and hydrophobic domain will appear outside of BSA folding surface. Therefore, BSA molecules contact with hydrophobic domain on material under shear stress. The hydrophobic domain will adsorb strongly on UHMWPE or CoCrMo alloy surface compared with hydrophilic part. The adsorption strength of denatured BSA molecule under shearing condition will be stronger compared with that of static adsorption. The potential did not dropped again except for immediate after first rubbing shown in Fig. 3, where BSA adsorbed strongly without desorption totally, while local adsorption and desorption occurs. Therefore, adsorbed BSA film under shear stress showed strong and stable. It is considered from Figs. 2 and 3 that the readsorption and reconstruction of BSA film is finished until $2000 \mathrm{~s}$, because the friction and potential behavior had become stable. The stability of potential was evaluated from the potential transition in PBS, and the trend in BSA solution was similar to the PBS results after $2000 \mathrm{~s}$.

Figure 4 indicates that BSA concentration affects on friction property. High concentration showed high friction. It is supposed that excessively adsorbed protein film increased friction due to the interaction. As described above, adsorbed BSA film under shear stress has high adsorption strength. Therefore difference in coefficient of friction is caused by changes in the structure and adsorption amount of BSA film, strong thin

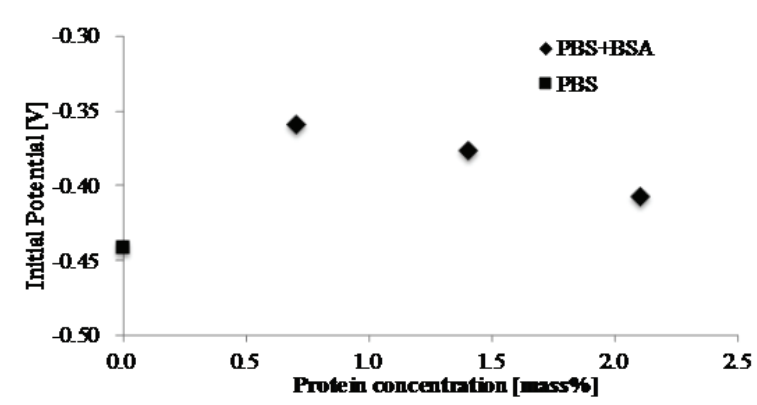

Fig. 8 Relationship between protein concentration in lubricant and initial potential under OCP condition. Initial potential decreased with BSA concentration

film increases friction coefficient under boundary lubrication regime. And it must take considering that surface coverage of BSA film clearly affects tribological property, because surface coverage affects direct contact of UHMWPE and CoCrMo alloy. High BSA concentration showed high potential drop shown in Fig. 5. The high potential drop indicates much BSA molecules desorption, and is affected by static adsorption before rubbing. Therefore, this fact suggests that high protein concentration formed much adsorption amount or thick film but weak adsorbed film. Most of desorbed BSA molecules at first rubbing had statically adsorbed before rubbing. Therefore, static adsorption is important to clarify the behavior of BSA desorption. The first static adsorption before rubbing was multilayer or high surface coverage under monolayer. Figure 8 shows initial potential which is recorded before starting of rubbing under OCP condition. The initial potential is affected by static adsorption of BSA molecules. Initial potential was decreased with BSA concentration. If surface coverage ratio became $100 \%$, initial potential should be constant. In this study, initial potential changed with BSA concentration. Therefore, the surface coverage is not saturated, so that BSA adsorption was monolayer and high concentration induced an increase in surface coverage. The difference of coefficient of friction depended on BSA surface coverage. The strong BSA film area increased with sliding distance under high concentration condition due to surface coverage increasing and as a result, shear resistance of BSA film was increased.

The trend of coefficient of friction under applied potential condition was similar to that under OCP condition, but coefficient of friction was low. The similar trend to OCP condition indicates that adsorbed BSA film was reconstructed by shear force during rubbing, as described above. The low coefficient of friction indicates the structure of adsorbed film or surface coverage is different from OCP condition. The CoCrMo alloy surface was charged $-0.2 \mathrm{~V}$, but OCP condition was under $-0.44 \mathrm{~V}$ during rubbing. The electric charge of surface property affects adsorption of proteins, so that 
adsorption in applied potential condition was different from OCP condition. Considering from the low coefficient of friction compared to that under OCP condition, adsorption amount under applied potential condition was small compared with that under OCP condition. BSA charges negative in $\mathrm{pH} 7.4$ solvent, it is considered that BSA molecules were repulsed electrically before rubbing under applied condition.

The current indicates amount of BSA desorption by rubbing. The current in BSA solution was high compared with that in PBS from starting of rubbing to $4000 \mathrm{~s}$. The high current indicates that adsorbed BSA molecules desorbed from the surface. Current peak was observed immediately after starting rubbing. The first rubbing sheared static adsorbed BSA molecules, and BSA molecules were desorbed by the first rubbing due to weak adsorption strength. After that, the current decreases as shown in Fig. 7, but it was still high compared with that in PBS. The static adsorption of BSA is rapid, finished within a second. The relative motion between CoCrMo alloy disk and UHMWPE pin was reciprocating, stationary CoCrMo alloy surface would statically adsorb BSA molecules in an interval of UHMWPE pin motion. The readsorbed BSA molecules in an interval sheared again, but desorption amount was small compared with first rubbing. If surface property was same before rubbing, same current should be observed. It is considered that static readsorption domain or adsorption strength had changed due to surface property change. As a result, desorption amount was changed. The surface property in this study is changed by BSA molecule adsorption. Therefore, adsorbed BSA molecules under shear force has denatured, and formed different adsorption film from static adsorption, then, surface property of CoCrMo alloy had changed. Therefore, transition of current in BSA solution indicates that first rubbing made BSA molecules desorbed, but not all BSA molecules. Remained BSA molecules on the surface denatured by shear force, and changed surface property. Finally, the current in BSA solution after $4000 \mathrm{~s}$ was close to that in PBS. The behavior indicates that adsorbed BSA film constructed under shear was stable, strong and had wear resistance for rubbing due to the formation under shear force. Therefore, it is concluded that the structure of BSA molecules on CoCrMo alloy under shear stress is reconstructed and had optimum structure for shear force after receiving shear force.

Considering the static adsorption behavior before rubbing, it is supposed that the adsorbed BSA film constructed under rubbing has different structure and property.

Periprosthetic synovial fluid contains not only albumin, but also other proteins, lipids and hyaluronic acid. Other constituents will have different effect on tribological property due to different adsorption behavior Especially, HGG has different conformation from albumin, and coexistence of albumin and HGG forms excellent boundary film [12]. The adsorption behavior and effect on tribological property is going to be investigated in future work.

\section{Conclusion}

The in-situ observation of adsorbed BSA film behavior during rubbing was carried out using electrochemical method. Adsorption and desorption behaviors of BSA molecule were observed during rubbing. BSA film on CoCrMo alloy surface was reconstructed during rubbing, and it became stable, strong and optimally-adapted structure for shear force by receiving shear force.

\section{Acknowledgement}

The part of this study received financial support given by the Grant-in-Aid for Specially Promoted Research of Japan Society for the Promotion of Science (23000011).

\section{References}

[1] Nakashima, K., Sawae, Y. and Murakami, T., "Friction Properties of the Protein Boundary Film Adsorbed on UHMWPE," Japanese Journal of Clinical Biomechanics, 31, 2010, 193-198 (in Japanese).

[2] Sawae, Y., Yamamoto, A. and Murakami, T., "Influence of Protein and Lipid Concentration of the Test Lubricant on the Wear of Ultra High Molecular Weight Polyethylene," Tribology International, 41, 7, 2008, 648-656.

[3] Costa, L., Luda, M. P., Trossarelli, L., Brach del Prever, E. M., Crova, M. and Gallinaro, P., "In Vivo UHMWPE Biodegradation of Retrieved Prosthesis," Biomaterials, 19, 15, 1998, 1371-1385.

[4] Eyerer, P. and Ke, Y. C., "Property Changes of UHMW Polyethylene Hip Cup Endoprostheses during Implantation," Journal of Biomedical Materials Research, 18, 9, 1984, 1137-1151.

[5] Valero Vidal, C. and Igual Munoz, A., "Study of the Adsorption Process of Bovine Serum Albumin on Passivated Surfaces of CoCrMo Biomedical Alloy," Electrochimica Acta, 55, 28, 2010, 8445-8452.

[6] Hiromoto, S. and Mischler, S., "The Influence of Proteins on The Fretting-Corrosion Behaviour of a Ti6Al4V Alloy," Wear, 261, 9, 2006, 1002-1011.

[7] Roba, M., Naka, M., Gautier, E., Spencer, N. D. and Crockett, R., "The Adsorption and Lubrication Behavior of Synovial Fluid Proteins and Glycoproteins on The Bearing-Surface Materials of Hip Replacements," Biomaterials, 30, 11, 2009 , 2072-2078.

[8] Yarimitsu, S., Nakashima, K., Sawae, Y. and Murakami, T., "Influences of Lubricant Composition on Forming Boundary Film 
Composed of Synovia Constituents," Tribology International, 42, 11-12, 2009, 1615-1623.

[9] Mishina, H. and Kojima, M., "Changes in Human Serum Albumin on Arthroplasty Frictional Surfaces," Wear, 265, 5-6, 2008, 655-663.

[10] Heuberger, M. P., Widmer, M. R., Zobeley, E., Glockshuber, R. and Spencer, N. D., "Protein-Mediated Boundary Lubrication in Arthroplasty," Biomaterials, 26, 2005, 1165-1173.

[11] Nakashima, K., Sawae, Y. and Murakami, T., "Study on Wear Reduction Mechanisms of Artificial Cartilage by Synergistic Protein Boundary Film Formation," JSME International Journal, 48, 4, 2005, 555-561.

[12] Nakashima, K., Sawae, Y. and Murakami, T., "Effect of Conformational Changes and Differences of Proteins on the Frictional Properties of Poly (Vinyl Alcohol) Hydrogel," Tribology International, 40, 10-12, 2007, 1423-1427.
[13] Nakashima, K., Sawae, Y. and Murakami, T., "Influence of Protein Conformation on Frictional Properties of Poly (Vinyl Alcohol) Hydrogel for Artificial Cartilage," Tribology Letters, 26, 2, 2007, 145-151.

[14] Igual Munoz, A. and Mischler, S., "Interactive Effects of Albumin and Phosphate Ions on the Corrosion of CoCrMo Implant Alloy," Journal of the Electrochemical Society, 154, 10, 2007, C562-C570.

[15] Mischler, S. and Igual Munoz, A., "Wear of CoCrMo Alloys Used in Metal-On-Metal Hip Joints: A Tribocorrosion Appraisal," Wear, 297, 1-2, 2013, 1081-1094.

[16] Mischler, S., "Sliding Tribocorrosion of Passive Metals: Mechanisms and Modeling in Tribo-Corrosion: Research, Testing and Applications," P. J. Blau et al. Editors, ASTM International, West Conshohocken, STP 1563, 2013, 1-18. 